\title{
LeGes
}

Katja Gfeller / Andreas Glaser / Irina Lehner

\section{E-Collecting: Umsetzungsvarianten und Rechtsetzungsbedarf}

Die Unterstützungsbekundung für Volksreferenden und Volksinitiativen bedingt eine handschriftliche Unterschrift. Die elektronische Sammlung von Unterschriften (E-Collecting) wurde im Zuge der Digitalisierung wiederholt diskutiert, ist bislang aber nicht zugelassen. Die Einschränkungen der Unterschriftensammlung aufgrund der Corona-Pandemie verleihen den rechtspolitischen Forderungen nach Einführung des E-Collecting neuen Auftrieb. Der Beitrag beleuchtet die Ausgestaltung der Unterstützungsbekundung durch einen elektronischen Identitätsnachweis, zeigt den daraus folgenden Rechtsetzungsbedarf auf und schliesst mit einer rechtspolitischen Einordnung.

Beitragsart: Werkstattberichte

Zitiervorschlag: Katja Gfeller / Andreas Glaser / Irina Lehner, E-Collecting: Umsetzungsvarianten und Rechtsetzungsbedarf, in: LeGes 32 (2021) 1 


\section{Inhaltsübersicht}

1. E-Collecting: Die Unterschriftensammlung der Zukunft?

1.1. Analoge Unterschriftensammlung als gesetzliches Leitbild

1.2. Einschränkung der Volksrechte während der Coronapandemie

1.3. Begriff und Verfahren des E-Collecting

2. Umsetzungsvarianten von E-Collecting

2.1. Eigenhändiges Unterzeichnen auf Touchscreen

2.1.1. Funktionsweise und Rechtsetzungsbedarf

2.1.2. Rechtspolitische Würdigung

2.2. Unterstützungsbekundung mit elektronischem Identitätsnachweis

2.2.1. Funktionsweise

2.2.1.1. Identifikation mittels E-ID bei Login

2.2.1.2. Login bei einer Anwendung

2.2.1.3. Sammlung der Unterstützungsbekundungen

2.2.1.4. Erteilung der Stimmrechtsbescheinigung

2.2.2. Verfassungsrechtliche und rechtspolitische Beurteilung

2.2.2.1. Sicherheit und Identifizierung

2.2.2.2. Stimmgeheimnis und Datenschutz

2.2.2.3. Effizienzgewinne

2.2.2.4. Verbreitung und Praktikabilität

2.2.3. E-ID oder anderer Identitätsnachweis?

2.2.4. Fazit

3. Rechtsetzungsbedarf

3.1. Verfassungsstufe

3.2. Gesetzesstufe

4. Rechtspolitische Herausforderungen

4.1. Überforderung des politischen Systems?

4.2. Deckelung des Anteils elektronisch gesammelter Unterschriften?

\section{E-Collecting: Die Unterschriftensammlung der Zukunft?}

\subsection{Analoge Unterschriftensammlung als gesetzliches Leitbild}

[1] Die gesetzlichen Regelungen zur Unterschriftensammlung für Referenden und Initiativen in Bund und Kantonen sind seit der Einführung dieser Volksrechte im 19. Jahrhundert auf analoge Vorgänge ausgerichtet [vgl. Art. 5 ff. BG vom 17. Juni 1874 betreffend Volksabstimmung über Bundesgesetze und Bundesbeschlüsse (AS 1 116); Art. 3 ff. BG vom 27. Januar 1892 über das Verfahren bei Volksbegehren und Abstimmungen betreffend Revision der Bundesverfassung (AS 12 885); heute Art. 60 ff. und Art. 68 ff. BG vom 17. Dezember 1976 über die politischen Rechte (BPR; SR 161.1)]. Zu sammeln sind eigenhändige in schriftlicher Form erteilte Unterschriften der Stimmberechtigten auf Papierbogen (Art. 60 und Art. 68 BPR). Zugelassen ist einzig die elektronische Zurverfügungstellung von Unterschriftenlisten, die heruntergeladen und ausgedruckt werden müssen, wobei keine Erleichterung hinsichtlich der Formerfordernisse gewährt wird (vgl. Art. 60a und Art. 69a BPR). Die ausgefüllten Unterschriftenbogen sind anschliessend bei der Gemeinde, in welcher die Unterzeichnenden stimmberechtigt sind, zur Beglaubigung (auch Bescheinigung) einzureichen (Art. 62 und Art. 70 BPR). Die Gemeinde gibt die beglaubigten Unterschriftenbogen den Absendern unverzüglich zurück (Art. 62 Abs. 2 und Art. 70 BPR), welche sie alsdann vor Ablauf der Sammelfrist bei der Bundeskanzlei bzw. auf kantonaler Ebene bei der Staatskanzlei zur Auszählung einreichen (vgl. Art. 66 und Art. 71 BPR). 
[2] Die Schaffung gesetzlicher Grundlagen für die Digitalisierung dieses Prozesses auf Bundesebene war bereits im Jahr 2008 mit der Motion 08.3908 Fehr «Stärkung der Demokratie durch E-Collecting» (Motion Fehr) angeregt worden. E-Collecting war als dritte Etappe in der Umsetzung von Vote électronique geplant (Bericht Vote électronique 2013, 5091 f.). Der Bundesrat hatte dementsprechend eine Rechtsgrundlage zur Erteilung einer Genehmigung an die Kantone «für Versuche zur Unterzeichnung eidgenössischer Volksbegehren auf elektronischem Wege» erlassen [Art. 27q V vom 24. Mai 1978 über die politischen Rechte (VPR; SR 161.11)], ohne dass jedoch davon Gebrauch gemacht wurde. Im Jahr 2017 wurde das Projekt der elektronischen Unterschriftensammlung vom Bund «angesichts knapper Ressourcen» vorläufig sistiert (Medienmitteilung 2017). In der Stellungnahme zur Motion 18.3062 Grüter vom 16. Mai 2018 «Stärkung der Volksrechte. Unterschriftensammlung für Initiativen und Referenden im Internet» führte der Bundesrat aus, es bestünden für die Einführung der elektronischen Unterschriftensammlung noch keine geeigneten Lösungen. Grundsätzlich sei mit dem Sistierungsentscheid aber nicht auf E-Collecting verzichtet worden. Es erscheine als angezeigt, so der Bundesrat in seinem jüngsten Civic-Tech-Bericht vom 8. Mai 2020, zunächst auf kommunaler und kantonaler Ebene Erfahrungen mit E-Collecting zu sammeln, bevor eine Einführung auf Bundesebene erwogen werde (Bericht Civic Tech, 23).

\subsection{Einschränkung der Volksrechte während der Coronapandemie}

[3] Infolge der Coronaviruspandemie wurde das Sammeln von Unterschriften für Referenden und Initiativen im öffentlichen Raum und die Zurverfügungstellung von Unterschriftenlisten vom 21. März bis 31. Mai 2020 vom Bundesrat schweizweit untersagt, auch ohne direkte Interaktion mit möglichen Unterzeichnern wie beispielsweise über die Plattform wecollect.ch [Art. $3 \mathrm{~V}$ vom 20. März 2020 über den Fristenstillstand bei eidgenössischen Volksbegehren (VFeV; AS 2020 847); Medienmitteilung 2020; zur Verfassungswidrigkeit der Verordnung vgl. Biaggini 2020, $227 \mathrm{ff}$.$] . Die Gemeinden wurden sodann aufgerufen, die Ausstellung von Stimmrechtsbeschei-$ nigungen vorübergehend einzustellen (Art. $4 \mathrm{Abs.} 1 \mathrm{VFeV}$ ).

[4] Aufgrund der seit Ausbruch der Pandemie geltenden Distanzregeln und der Zurückhaltung der Menschen blieb es indes auch nach Ausserkrafttreten des Verbots faktisch sehr schwierig, Unterschriften für Referenden und Initiativen zu sammeln. Darin dürfte der Grund liegen für das erneute Aufflammen der Diskussion um die Einführung der elektronischen Unterschriftensammlung (vgl. z. B. von Matt 2020; Gerny 2020). In einzelnen Kantonen wird die Schaffung der für E-Collecting erforderlichen Rahmenbedingungen daher wieder stärker vorangetrieben (so insbesondere mit der Motion Schaffhausen; zuvor auch in St. Gallen, vgl. Interpellation Louis/Schmid). Rechtliche Grundlagen für eine Digitalisierung der Unterschriftensammlung, die über das Zurverfügungstellen von elektronischen Unterschriftenlisten hinausgehen, existieren bisher aber noch nirgendwo.

[5] Die pandemiebedingten Schwierigkeiten beim Sammeln von Unterschriften auf der Strasse und die daraus resultierende Lähmung der direktdemokratischen Rechte könnten als politische Katalysatoren für die Beschleunigung der Zulassung der elektronischen Unterschriftensammlung wirken. 


\subsection{Begriff und Verfahren des E-Collecting}

[6] E-Collecting bezeichnet die Unterzeichnung von Volksbegehren in Form von Referenden und Initiativen auf elektronischem Wege (vgl. Art. 27q VPR). Auch die rechtspolitischen Vorstösse in den Kantonen beziehen sich auf die direktdemokratischen Instrumente (exemplarisch Motion Schaffhausen). Auf längere Sicht könnte als weitere Etappe die elektronische Unterzeichnung von Wahlvorschlägen hinzukommen (Bericht Vote électronique 2013, 5092). Im Folgenden wird indes nur auf die gegenwärtig im Vordergrund stehende Unterschriftensammlung für Referenden und Initiativen eingegangen.

[7] Das Unterschreiben respektive die Unterstützungsbekundung in digitaler Form gliedert sich in vier Teilprozesse: Als erstes die Abgabe der Unterstützungsbekundung durch die stimmberechtigte Person, zweitens das Sammeln der Unterstützungsbekundungen durch die Komitees, drittens die Überprüfung der Gültigkeit der Unterstützungsbekundungen durch die Bescheinigungsstellen (Gemeinden) und zuletzt das Zählen der gültig abgegebenen Unterstützungsbekundungen durch eine zentrale staatliche Stelle, d. h. die Staats- resp. Bundeskanzlei (Bericht Civic Tech, 22). Dabei entstehen Schnittstellen zwischen diesen Teilprozessen und den jeweils dafür zuständigen Akteuren, so beispielsweise die Übermittlung der durch die Komitees gesammelten Unterstützungsbekundungen an die Gemeinden zwecks Bescheinigung (vgl. Bericht Civic Tech, 23).

[8] Auch Vorgänge, bei denen nicht alle vier Schritte elektronisch ablaufen, können als E-Collecting angesehen werden. Voraussetzung ist aber, dass die Unterstützungsbekundung selbst (erster Schritt) digital erfolgt, wodurch die heute zulässige Variante, bei der elektronische Unterschriftenlisten zur Unterzeichnung von den Stimmberechtigten ausgedruckt und dem Sammelkomitee per Post zugestellt werden müssen (vgl. Art. 60a und Art. 69a BPR und nunmehr Bundeskanzlei 2020), nicht darunterfällt.

\section{Umsetzungsvarianten von E-Collecting}

[9] Im Zentrum der nachfolgenden Ausführungen steht die Umsetzungsvariante der Unterstützungsbekundung mit elektronischem Identitätsnachweis (siehe Ziff. 2.2). Vorab soll überblicksartig auf die Variante des eigenhändigen Unterzeichnens auf Touchscreens eingegangen werden, zumal die Zulassung dieser Variante im Nachgang zur ersten Coronaviruswelle gefordert wurde (vgl. Stiftung für direkte Demokratie 2020).

[10] Die Möglichkeit, einen elektronischen Unterschriftenbogen mittels «Anheftens» einer qualifizierten elektronischen Signatur im Sinne des Bundesgesetzes vom 18. März 2016 über die elektronische Signatur (ZertES; SR 943.03) zu unterzeichnen, wird nachfolgend nicht vertieft. Zum einen haben qualifizierte elektronische Signaturen unter Privatpersonen bislang kaum Verbreitung gefunden, obwohl sie bereits seit einiger Zeit auf dem Markt angeboten werden. Zum anderen fehlt eine Verknüpfung der qualifizierten elektronischen Signatur mit der Stimmberechtigung, sodass eine automatisierte Überprüfung der Stimmberechtigung nicht möglich wäre, weshalb die elektronische Unterschrift kaum als sinnvolle Lösung zum Identitätsnachweis betrachtet werden könnte. 


\subsection{Eigenhändiges Unterzeichnen auf Touchscreen}

\subsubsection{Funktionsweise und Rechtsetzungsbedarf}

[11] Die elektronische Unterschriftensammlung durch Unterzeichnung auf Touchscreens ist gewissermassen eine direkte Übertragung des heutigen analogen Systems auf den digitalen Weg. Anstatt auf Papier unterzeichnen die Stimmberechtigten mit dem Finger oder einem Stift auf einem Touchscreen einen digitalen Unterschriftenbogen. Der «elektronisch» unterzeichnete Unterschriftenbogen wird (von der Einzelperson oder dem Sammelkomitee) ausgedruckt und der Gemeinde zur amtlichen Bescheinigung zugesandt. Allenfalls wäre eine elektronische Übermittlung (als pdf) von den Unterzeichnenden an die Gemeinde denkbar. Die bescheinigten Unterschriftenbogen werden anschliessend dem Komitee zugestellt. Möglich wäre allenfalls auch hier eine direkte elektronische Übermittlung an die Bundes- bzw. Staatskanzlei.

[12] Bei dieser Umsetzungsvariante stellt sich in erster Linie die Frage, ob eine gesetzliche Grundlage zu schaffen wäre oder ob sich das eigenhändige Unterzeichnen auf Touchscreens unter die auf Bundesebene und in den meisten Kantonen bestehende Formvorschrift des eigenhändigen Unterzeichnens subsumieren liesse [Bund: Art. 61 Abs. 1 und Art. 70 BPR; Kantone statt vieler z. B. Aargau: § 43 Abs. 1 und § 62a Abs. 1 Gesetz über die politischen Rechte vom 10. März 1992 (SAR 131.100); Genf: Art. 87 Abs. 2 Loi sur l'exercice des droits politiques vom 15. Oktober 1983 (RSG A 5 05)]. Eine Auslegung des Formerfordernisses der Eigenhändigkeit ergibt keine klare Antwort. Der Wortlaut würde grundsätzlich eine Gleichstellung von eigenhändiger Unterschrift auf Touchscreens und eigenhändiger Unterzeichnung auf Papier erlauben. Auch Sinn und Zweck der Formvorschrift (Identifikation) sprächen nicht dagegen.

[13] Aus historischer Sicht kann mit der Eigenhändigkeit mangels digitaler Möglichkeiten im Zeitpunkt des Erlasses der Vorschriften jedoch nur die Unterzeichnung auf Papier gemeint gewesen sein. Die auf Bundesebene erst kürzlich erfolgte Ergänzung der Formvorschrift, wonach auch Name und Vorname handschriftlich und leserlich auf die Liste geschrieben werden müssen, spricht ebenfalls gegen eine weite Auslegung des Eigenhändigkeitserfordernisses [vgl. BPR, Änderung vom 26. September 2014 (AS 2015 543)]. Insgesamt herrscht in Bezug auf die Beurteilung der Rechtsgültigkeit einer Unterschrift auf einem Touchscreen somit jedenfalls erhebliche Rechtsunsicherheit. Es kann den Sammelnden nicht empfohlen werden, sich auf die Gültigkeit von über Touchscreens gesammelten Unterschriften zu verlassen. Für die rechtssichere Zulassung von Unterschriften auf Touchscreens bei der Unterschriftensammlung für Referenden und Initiativen wäre eine gesetzliche Bestimmung zu erlassen, wonach die eigenhändige Unterschrift auf einem Touchscreen derjenigen auf Papier gleichgestellt ist.

\subsubsection{Rechtspolitische Würdigung}

[14] Der Vorteil dieses Verfahrens besteht aus praktischer Sicht darin, dass mobile Geräte mit Touchscreen weit verbreitet und die Bürgerinnen und Bürger mit ihrer Handhabe gut vertraut sind (GFeller et al. 2019, 56), auch wenn dies noch nicht für das Unterschreiben auf einem Touchscreen anstatt auf Papier gilt. Initiativen und Referenden könnten von den Stimmberechtigten aber jedenfalls ohne grossen Aufwand «von zu Hause aus» unterstützt werden, was gerade in Zeiten von Corona zu begrüssen wäre. Allerdings lassen das Ausdrucken der elektronisch unterzeichneten Unterschriftenbogen und die weiterhin manuell vorzunehmende Bescheinigung der Unterschriften durch die Gemeinden den Prozess als schwerfällig erscheinen. Abgesehen von 
den Erleichterungen für die Sammelkomitees sind - gerade auf Seiten der Behörden - kaum Effizienzgewinne zu erwarten. Der Aufwand für die Schaffung von rechtlichen Grundlagen für eine rechtssichere Zulassung von auf einem Touchscreen unterzeichneten Unterstützungsbekundungen wäre vor diesem Hintergrund unverhältnismässig und die Umsetzungsvariante wenig zukunftsträchtig.

\subsection{Unterstützungsbekundung mit elektronischem Identitätsnachweis}

\subsubsection{Funktionsweise}

[15] Betreffend die Abgabe der Unterstützungsbekundung ist bei dieser Umsetzungsvariante einerseits festzulegen, wie der Identitätsnachweis zu erbringen ist. Andererseits ist die Anwendungsplattform zu definieren, über die sich die Person identifiziert.

\subsubsection{Identifikation mittels E-ID bei Login}

[16] Die Feststellung der Identität soll via Login-Verfahren erfolgen. Dabei kommt die Verwendung einer E-ID oder eines anderen Verfahrens unter Nutzung eines sogenannten eindeutigen Personenidentifikators wie bspw. die nichtsprechende AHV-Nummer (AHVN13) (siehe dazu Ziff. 2.2.2.1) als Identitätsnachweis in Frage (zur Wahl zwischen beiden Varianten siehe 2.2.3).

[17] Die E-ID ist eine Möglichkeit, die eigene Identität im elektronischen Geschäftsverkehr mit Behörden (E-Government) und Privaten eindeutig und rechtsgültig auszuweisen (Botschaft BGEID 2018, 3916). Auf Bundesebene lag ein vom Parlament angenommener Entwurf zu einem Bundesgesetz über die E-ID vor [Entwurf BG über elektronische Identifizierungsdienste vom 27. September 2019, BBl 20196567 (E-BGEID)]. Der Gesetzesentwurf wurde in der Volksabstimmung vom 7. März 2021 klar abgelehnt. Es ist indes zu erwarten, dass auch in künftigen Gesetzgebungsprojekten an der im E-BGEID beschriebenen, eigentlichen Funktionsweise der E-ID festgehalten wird, weshalb diese nachfolgend in ihren Grundzügen nachgezeichnet wird.

[18] Jede Person kann bei der zuständigen Behörde eine E-ID beantragen, wobei ein amtlicher Ausweis zur Identifikation vorzulegen ist. Die Person erhält von der Behörde alsdann eine E-ID-Registrierungsnummer, unter der bestimmte, in den verschiedenen Registern gespeicherte Personenidentifizierungsdaten der antragstellenden Person, z. B. Name, Geburtsdatum und Gesichtsbild, gespeichert werden. Zur eindeutigen Identifizierung der antragstellenden Person könnte die nichtsprechende AHV-Nummer als eindeutiger Personenidentifikator verwendet werden. Die antragstellende Person erhält den Zugang zur Nutzung ihrer E-ID mittels eines $\mathrm{Au}-$ thentifizierungsmittels (i.d.R. mobiles Gerät), mit dem sie sich - in Verbindung mit einem persönlichen Passwort - online ausweisen kann. Die einmal auf der E-ID gespeicherten Personenidentifizierungsdaten werden weiterhin periodisch bei den staatlichen Registern abgefragt und nötigenfalls aktualisiert.

[19] Bei der Verwendung der E-ID zur Unterstützung von Volksbegehren müsste die Stimmberechtigung ebenfalls auf der E-ID «gespeichert» und vor jeder Nutzung erneut bei der Gemeinde abgefragt werden. So könnte die Unterzeichnung von Volksbegehren durch E-ID-Nutzer ohne Stimmberechtigung von vornherein verhindert werden. 


\subsubsection{Login bei einer Anwendung}

[20] Es stellt sich die Frage, auf welcher Anwendung oder Plattform das Login zwecks elektronischer Unterstützungsbekundung erfolgen soll. Denkbar sind die Verknüpfung einer E-CollectingAnwendung mit E-Government-Angeboten, eine von E-Government unabhängige, aber «zentralisierte» Anwendung oder einzelne Plattformen für jedes Initiativ- und Referendumsbegehren. Die technische Umsetzung all dieser Möglichkeiten könnte in Form von Webseiten oder Applikationen erfolgen.

[21] Die erste Weichenstellung erfolgt bei der Frage, ob die Sammlung staatlich oder privat organisiert wird. Eine zentrale Anwendung wäre wohl, ebenso wie das E-Government-Portal, staatlich zu betreiben. Einzelne Plattformen hingegen würden von Privaten (von den Sammelkomitees oder, aufgrund der dafür nötigen technischen Kenntnisse und Infrastruktur wahrscheinlicher, von Dritten) aufgeschaltet. Da bei Referenden im Unterschied zu Initiativen nicht notwendigerweise ein formelles Komitee besteht (vgl. Art. 60 Abs. 1 und Art. 68 Abs. 1 Bst. e BPR) ist eine zentrale Lösung aus Praktikabilitätsgründen naheliegend.

[22] E-Government-Portale sind auf der kantonalen Ebene bereits weit verbreitet und werden laufend ausgebaut (E-Government Schweiz 2019, 19). Auf diesen Portalen können Bürgerinnen und Bürger verschiedenste Angebote der Behörden online in Anspruch nehmen, die früher «am Schalter» erledigt werden mussten, beispielsweise das Einreichen der Steuererklärung, Umzugsmeldungen oder Anträge für Stipendien. Eine Anwendung zur elektronischen Unterstützung von Referenden oder Initiativen könnte somit an die bestehenden kantonalen E-Government-Portale angegliedert werden.

\subsubsection{Sammlung der Unterstützungsbekundungen}

[23] Die eingereichten Unterstützungsbekundungen müssen heute an zentraler Stelle gesammelt werden, damit sie anschliessend durch die Behörden auf ihre Gültigkeit überprüft werden können. Es ist fraglich, ob es weiterhin nötig wäre, elektronische Unterstützungsbekundungen zunächst zentral (bspw. beim Komitee) zu sammeln, bevor sie zur Überprüfung an die Behörden weitergeleitet werden (Bericht Civic Tech, 22). Entfällt dieser Schritt künftig, müsste allerdings weiterhin gewährleistet werden, dass die Komitees während der laufenden Sammelfrist über den Stand der Anzahl eingereichter Unterstützungsbekundungen unterrichtet sind. Den Komitees muss zudem die Möglichkeit der Nichteinreichung oder des Rückzugs ihrer Initiative erhalten bleiben (vgl. Art. 73 BPR).

\subsubsection{Erteilung der Stimmrechtsbescheinigung}

[24] Für ein medienbruchfreies E-Collecting muss die Stimmberechtigung der unterzeichnenden Person auf elektronischem Weg überprüft werden. Dadurch kann der Bescheinigungsprozess automatisiert erfolgen, sodass die manuelle Überprüfung des Stimmrechts und der Formvorschriften entfällt (AmmanN/Schnell 2019, 21 und 24). Grundvoraussetzung dafür ist die elektronische Führung der Stimmregister. 


\subsubsection{Verfassungsrechtliche und rechtspolitische Beurteilung}

\subsubsection{Sicherheit und Identifizierung}

[25] Die Unterschriftensammlung für Volksinitiativen und Referenden ist von der Garantie der politischen Rechte nach Artikel 34 Absatz 1 der Bundesverfassung (BV; SR 101) umfasst (BGE 139 II 303 E.7.1). Zentral bei der Einführung von E-Collecting ist die Erfüllung der für die Unterschriftensammlung einschlägigen Sicherheitsanforderungen, um das rechtmässige Zustandekommen von Initiativen und Referenden gewährleisten zu können. Gemäss Artikel 27q VPR müssen alle wirksamen und angemessenen Massnahmen ergriffen werden, um die Kontrolle der Stimmberechtigung, das Stimmgeheimnis und die korrekte Zuordnung aller Unterschriften zu gewährleisten und um die Gefahr gezielten oder systematischen Missbrauchs ausschliessen zu können (vgl. auch EDÖB 2019, 10). Diese bundesrechtliche Vorgabe ist auf die Unterschriftensammlung für Volksinitiativen und Referenden auf kantonaler Ebene zwar nicht unmittelbar anwendbar, sie könnte jedoch als Orientierungshilfe betreffend Sicherheitsstandards herangezogen werden (vgl. Art. 39 Abs. 1 BV, wonach die Kantone die Ausübung der politischen Rechte in kantonalen und kommunalen Angelegenheiten regeln).

[26] Die Identitätsfeststellung für eine Unterstützungsbekundung verfolgt also im Wesentlichen zwei Ziele: einerseits die zuverlässige und eindeutige Identifizierung der das Begehren unterstützenden Personen, andererseits die Verhinderung von Identitätsdiebstahl und Mehrfachunterzeichnungen (vgl. Ammann/Schnell 2019, 21). Heute wird das Handschriftlichkeits- und Unterschriftserfordernis zum Erreichen beider Ziele eingesetzt, zusammen mit weiteren Angaben wie Geburtsdatum und Wohnadresse (vgl. Art. 61 BPR; siehe auch Ziff. 2.1.1). Beim E-Collecting mit elektronischem Identitätsnachweis entfällt die handschriftliche Unterschrift. Nichtsdestotrotz können beide Ziele auch bei dieser Umsetzungsvariante erreicht und so die notwendige Sicherheit gewährleistet werden.

[27] Für die elektronische Unterzeichnung drängt sich zur Identifizierung die Verwendung eines sogenannten eindeutigen Personenidentifikators auf. Es handelt sich dabei um eine Nummer, die der betreffenden Person unzweideutig zugeordnet werden kann. Beispielsweise ist die nichtsprechende AHV-Nummer (AHVN13) ein solcher eindeutiger Personenidentifikator. Durch die Nutzung eines solchen eindeutigen Personenidentifikators wäre es möglich, die Stimmberechtigung der betreffenden Person (vgl. Art. 62 Abs. 2 BPR) automatisiert und unzweifelhaft zu überprüfen.

[28] Weiter müssen Unterstützungsbekundungen unter falschem Namen (sog. Identitätsdiebstahl) verhindert werden (vgl. Art. 27q VPR; vgl. auch EDÖB 2019, 10). Als Mindeststandard für eine sichere elektronische Unterstützungsvariante, die Identitätsdiebstahl verunmöglicht, ist die Zwei-Faktoren-Authentifizierung anzusehen. Diese ist den Anwendern bereits aus anderen Bereichen mit hohen Sicherheitsanforderungen bekannt, beispielsweise dem E-Banking (Bisaz/ SERDÜlt 2017, 534).

[29] Schliesslich müssten Mehrfachunterstützungen durch dieselbe stimmberechtigte Person in Form von digitalen sowie analogen Unterstützungsbekundungen (BISAZ/SERDÜLt 2017, 540; Braun Binder 2014, 551) oder bei einem Wohnsitzwechsel verunmöglicht werden (siehe zu einem Fall doppelter elektronischer Stimmabgabe nach einem Wohnsitzwechsel BGer, Urteil vom 18. April 2018,6B_604/2017). Neben der Nutzung eines eindeutigen Personenidentifikators ist dafür die Harmonisierung (mit Zugriff der übergeordneten Staatsebenen auf die untergeordneten Register) oder Zentralisierung der Stimmregister Voraussetzung (BRAUn BINDER 2014, 549; 
siehe auch Stellungnahme des Bundesrats zur Motion Fehr; Stellungnahme des Bundesrats zur Interpellation Wermuth). ${ }^{1}$ So könnte das Risiko von Fälschungen und Mehrfachunterzeichnungen gegenüber heute deutlich gesenkt werden (Ammann/Schnell 2019, 21).

[30] Als weitere bestehende Massnahme gegen den Missbrauch dient der Warnhinweis auf die Strafbarkeit der Fälschung von Unterschriften (Art. 60 Abs. 1 Bst. c BPR). Dieser könnte prominenter als auf den Unterschriftenbogen platziert werden, etwa indem er als «Pop-Up-Fenster» in der Applikation oder auf der entsprechenden Website erscheint, dessen Kenntnisnahme durch einen Klick bestätigt werden müsste (BraUn Binder 2014, 554).

[31] Insgesamt sind weder das heutige manuelle System noch ein künftiges E-Collecting-System geeignet, jedes Manipulationsrisiko auszuschliessen (vgl. BisAz/Serdült 2017, 533 f.). Ein gewisses Missbrauchspotenzial bei der Unterschriftensammlung bleibt unvermeidbar (vgl. Bericht Vote électronique 2002, 664). Zwingend ist jedoch, dass beim E-Collecting mindestens der Sicherheitsstandard der manuellen Unterschriftensammlung beibehalten wird (Bericht Vote électronique 2002, 664; BRAUn Binder 2014, 552). Dies erscheint bei der Nutzung der E-ID möglich, die durch den eindeutigen Personenidentifikator zusammen mit der Zwei-Faktoren-Authentifizierung eine sicherere Identifikation zulässt als die analoge Methode (ähnlich Ammann/Schnell 2019, 21).

\subsubsection{Stimmgeheimnis und Datenschutz}

[32] Das Stimmgeheimnis ist grundsätzlich auch hinsichtlich Initiativ- und Referendumsunterschriften zu wahren (BGE 98 Ia 602 E.8; Braun 2006, Rz. 486). Diese Daten dürfen aber von den Sammelkomitees sowie den Behörden bearbeitet werden. Dem Stimmgeheimnis kommt also bei der Unterschriftensammlung nicht dieselbe überragende Bedeutung zu wie beim E-Voting (BISAz/Serdült 2017, 553). Bei der Abgabe der Unterstützungsbekundung auf elektronischem Weg wäre das Stimmgeheimnis wohl sogar besser geschützt als beim heutigen «manuellen» Unterschriftensammeln im öffentlichen Raum.

[33] Schliesslich sind beim E-Collecting die datenschutzrechtlichen Bestimmungen einzuhalten. Auf Private und Bundesbehörden ist das Bundesgesetz über den Datenschutz ${ }^{2}$ (DSG) anwendbar (vgl. Art. 2 Abs. 1 Bst. a DSG; ebenso Art. 2 Abs. 1 Bst. a E-DSG), auf Organe von Kantonen und Gemeinden die jeweilige kantonale Datenschutzgesetzgebung (vgl. EDÖB 2019, 3). Politische Ansichten stehen demnach unter erhöhtem Schutz, da es sich um «besonders schützenswerte Personendaten» gemäss Artikel 3 Buchstabe c Ziffer 1 DSG handelt (ebenso Art. 5 Bst. a Ziff. 1 E-DSG; siehe auch EDÖB 2019, 3 f.; Bericht Civic Tech, 23). Daher ist für deren Bearbeitung eine formell-gesetzliche Grundlage erforderlich (Art. 17 DSG; ähnlich Art. 34 E-DSG). Gegenüber heute wäre das Risiko des Missbrauchs der Daten aufgrund der elektronischen Verarbeitung wohl höher. Nichtsdestotrotz ist davon auszugehen, dass die heutigen datenschutzrechtlichen Bestimmungen genügen würden (vgl. auch die zusätzlichen Datenschutzbestimmungen in Art. 9 und Art. 15 f. E-BGEID).

1 Beispiele sind etwa der Kanton Solothurn, in dem für den Datenaustausch eine Stimmregisterplattform besteht, welche die von den Gemeinden geführten Register vernetzt [\$1 Abs. 3 Bst. a Gesetz vom 5. November 2014 über die Einwohnerregister- und die Stimmregisterplattform (BGS 114.3)], ähnlich der Kanton Obwalden [vgl. Art. 4 Gesetz vom 4. Dezember 2008 über die Harmonisierung der amtlichen Register (OGS 131.4)].

2 Heute Bundesgesetz vom 19. Juni 1992 über den Datenschutz (SR 235.1). Dessen Ersatz steht bevor: die Referendumsfrist des E-DSG ist am 14. Januar 2021 abgelaufen, der Bundesrat wird das Gesetz zur gegebenen Zeit in Kraft setzen [BG vom 25. September 2020 über den Datenschutz (Datenschutzgesetz, DSG2020, BB1 2020 7639)]. 


\subsubsection{Effizienzgewinne}

[34] Der Abgleich der Daten mit den Personenregistern (resp. den Stimmregistern) kann bei der E-ID automatisiert erfolgen (vgl. Art. 24 Abs. 3 E-BGEID), was den Behörden einen Grossteil der heute anfallenden Arbeiten bei der manuellen Stimmrechtsbescheinigung ersparen kann. Diese Automatisierung hat somit das Potenzial bedeutender Effizienzgewinne auf Seiten der Behörden (Ammann/Schnell 2019, 21). Damit könnte eine derartig ausgestaltete E-Collecting-Lösung das Effizienzversprechen nicht nur zugunsten der Stimmberechtigten und der Komitees (vgl. Bericht Civic Tech, 13; BisAz/SERDüLt 2017, 531), sondern auch zugunsten der staatlichen Stellen einlösen (vgl. Bericht Civic Tech, 8).

[35] Harmonisierte oder zentralisierte Stimmregister (siehe Ziff. 2.2.2.1) würden auch zu einer erhöhten Planungssicherheit für die Sammelkomitees führen, denn so könnten Mehrfachunterzeichnungen schneller festgestellt werden. Heute bedarf es hingegen einer gewissen Sicherheitsmarge an «überzähligen» Unterschriften, um erst später entdeckte Mehrfachunterzeichnungen zu kompensieren (BRAUn Binder 2014, 551).

\subsubsection{Verbreitung und Praktikabilität}

[36] Verschiedene Kantone bieten heute bereits das Login auf ihrem E-Government-Portal über eine E-ID an, so die Kantone Aargau, ${ }^{3}$ Bern, ${ }^{4}$ Jura, ${ }^{5}$ Schaffhausen, ${ }^{6}$ Solothurn, ${ }^{7}$ St. Gallen ${ }^{8}$ und Zug. ${ }^{9}$ Die Ausstellung der E-ID erfolgt im Kanton Schaffhausen bereits mittels einer Kontrolle des Eintrags im Einwohnerregister. Diese Verbreitung macht einerseits E-Government-Portale für eine weitergehende Anwendung als E-Collecting-Plattform attraktiv und kann Synergieeffekte herbeiführen. Andererseits ist E-Collecting im Sinne der Ausübung der direktdemokratischen Grundrechte doch von deutlich anderem Charakter als beispielsweise eine Umzugsmeldung. Diesem Umstand muss Rechnung getragen werden, wenn E-Collecting an eine E-GovernmentPlattform angegliedert werden sollte.

\subsubsection{E-ID oder anderer Identitätsnachweis?}

[37] Wie oben kurz erwähnt, wäre anstelle der E-ID die Nutzung eines alternativen Identitätsnachweises möglich. Voraussetzung dafür ist, dass dieser die Standards einer Zwei-FaktorenAuthentifizierung und eines eindeutigen Personenidentifikators erfüllt, beispielsweise mittels Rückgriffs auf die nichtsprechende AHV-Nummer (AHVN13). Unter der heutigen Rechtslage muss für die Nutzung der AHVN13 eine spezialgesetzliche Grundlage bestehen, welche die Verwendung der Nummer ausdrücklich erlaubt [Art. 50e Abs. 3 BG vom 20. Dezember 1946 über die Alters- und Hinterlassenenversicherung (AHVG; SR 831.10)]. Auf die Schaffung einer spezialgesetzlichen Regelung für die Verwendung der Nummer beim E-Collecting könnte indes verzich-

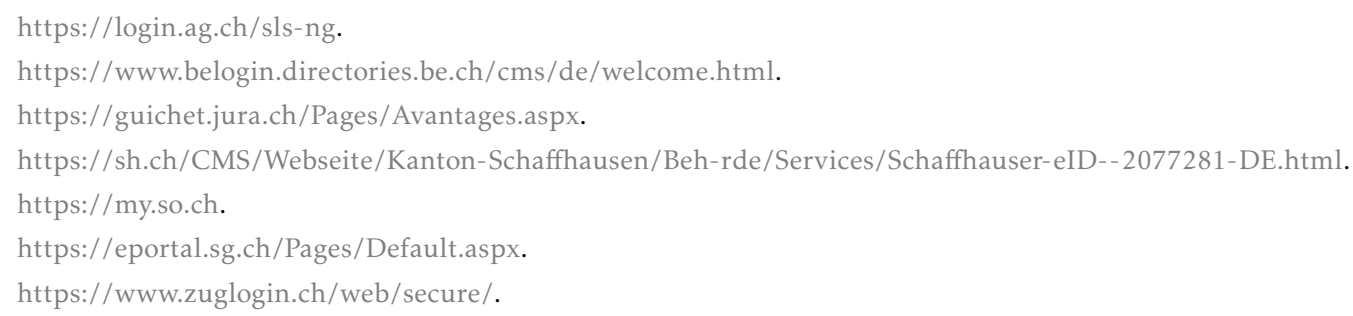


tet werden, sofern die vom Parlament in der Wintersession 2020 verabschiedeten gesetzlichen Grundlagen zur systematischen Verwendung der AHVN13 ausserhalb der AHV wirksam werden [vgl. dazu die Art. 153b-Art. 153g Entwurf geändertes BG über die Alters- und Hinterlassenenversicherung (E-AHVG; BBl 2020 9951) sowie Art. 20 Abs. 3 Entwurf Informationssicherheitsgesetz (E-ISG; BBl 2020 9975); Referendumsfrist jeweils bis zum 10. April 2021; siehe zum Ganzen auch Botschaft Änderung AHVG (BBl 2019 7361)].

[38] Letztlich dürfte aus Gründen der Praktikabilität gleichwohl die E-ID für das E-Collecting zu bevorzugen sein. Der Einsatz der E-ID beim E-Collecting profitiert von beträchtlichen Synergieeffekten mit anderen Nutzungen, was die Verbreitung in der Bevölkerung fördern sollte. Die Breite der geplanten Verwendungsmöglichkeiten deutet darauf hin, dass die E-ID auch für technisch nicht versierte Personen nutzbar sein wird (vgl. zu dieser Anforderung BRAUn Binder 2014, 549 m.w.H.). Insgesamt käme ein Einsatz der E-ID beim E-Collecting als eine von deren Kernfunktionen im Sinne einer sicheren, vertrauenswürdigen Identifizierung im Internet in Betracht (vgl. Botschaft BGEID 2018, 3916). Die rechtliche Ausgestaltung ist demnach hierauf auszurichten, gerade auch in Verbindung mit E-Government-Portalen, für welche die E-ID explizit einsetzbar sein soll (Botschaft BGEID 2018, 3924). Die Verwendung anderer Identifikationsmittel verspricht demgegenüber keinen vergleichbaren Mehrwert.

\subsubsection{Fazit}

[39] Es erscheint empfehlenswert, dass zuerst in einigen, betreffend Digitalisierung weit fortgeschrittenen Kantonen Versuche mit E-Collecting mittels E-ID auf E-Government-Portalen durchgeführt werden. $\mathrm{Zu}$ nennen ist beispielsweise der Kanton Schaffhausen, in dem bereits eine E-ID als Login für das E-Government-Portal genutzt wird. Diese E-ID wird mittels Abgleichs mit den elektronisch geführten Einwohnerregistern [Art. 13 Abs. 1 Wahlgesetz vom 15. März 1904 (SHR 160.100)] erstellt (Informatik Kanton und Stadt Schaffhausen 2019), womit sie eine wichtige Voraussetzung für das E-Collecting erfüllt. Im Wege einer Motion wird denn auch gefordert, auf Basis dieser E-ID das E-Collecting für kantonale Volksbegehren zu ermöglichen (Motion Schaffhausen).

\section{Rechtsetzungsbedarf}

\subsection{Verfassungsstufe}

[40] Die Umsetzung des E-Collecting mit elektronischem Identitätsnachweis bedingt eine Anpassung der Rechtsgrundlagen. Es stellt sich die Frage, ob hierzu eine Revision der Verfassung erforderlich ist. Auf Bundesebene enthalten die Bestimmungen zum fakultativen Referendum (Art. 141 BV) und zur Volksinitiative (Art. 138 und Art. 139 BV) keinerlei Vorgaben zur Art und Weise der Unterschriftensammlung; festgelegt sind jeweils nur die Zahl der Unterschriften und die Frist für die Unterschriftensammlung (anders aber Bisaz/Serdült 2017, 542). Vergleichbares gilt für die kantonale Ebene. Exemplarisch legt im Kanton St. Gallen gemäss Artikel $44 \mathrm{Absatz}$ 1 der Verfassung des Kantons St. Gallen (KV/SG; sGS 111.1) (Initiative) und Artikel 50 Absatz 2 KV/SG (Referendum) das Gesetz das Verfahren für das Lancieren bzw. Ergreifen von Initiativen und Referenden fest. Es steht dem Gesetzgeber mithin frei, die elektronische Unterschriftensammlung zuzulassen, ohne dass dafür Verfassungsänderungen notwendig sind. 


\subsection{Gesetzesstufe}

[41] Die Grundzüge des E-Collecting müssten auf der Stufe des Gesetzes mit hinreichender Bestimmtheit geregelt werden. Die grundlegenden Bestimmungen über die Ausübung der politischen Rechte (Art. 164 Abs. 1 zweiter Satz Bst. a BV) gehören zu den wichtigen rechtsetzenden Bestimmungen, die in der Form des Bundesgesetzes zu erlassen sind (Art. 164 Abs. 1 erster Satz BV); die Verordnungsebene genügt daher nicht. Betroffen ist ausserdem das Verfahren der Bundesbehörden (Art. 164 Abs. 1 zweiter Satz Bst. g BV). Das neue elektronische Sammelverfahren müsste auch in den Kantonen auf Gesetzesstufe geregelt werden, denn es werden dadurch - exemplarisch für den Kanton St. Gallen - einerseits die Rechte von Privaten berührt (vgl. Art. 67 Abs. 1 Bst. a KV/SG) und andererseits die Grundzüge des kantonalen Gesetzgebungsverfahrens geordnet (vgl. Art. 67 Abs. 1 Bst. b KV/SG). Weniger wichtige, z. B. vorwiegend technische Bestimmungen, könnten daneben der Verordnungsgebung überlassen werden.

[42] Erforderlich wäre auf Bundesebene in erster Linie eine Ergänzung von Artikel 61 BPR. Um zu ermöglichen, dass die E-ID zur Unterstützungsbekundung gültig genutzt werden kann, müsste die Bestimmung dahingehend angepasst werden, dass neben analogen Unterschriften (mit Handschriftlichkeitserfordernis) die Verwendung eines sicheren Logins respektive einer staatlich anerkannten E-ID ebenfalls zulässig wäre (vgl. Bericht Civic Tech, 22). Notwendig wären zudem Folgeänderungen in den Artikeln 60 ff. und 68 ff. BPR. Hinzu kommt die elektronische Führung der Stimmregister, die ebenfalls eine gesetzliche Grundlage bedingt (vgl. Art. 4 BPR). Allenfalls wäre dies auch über eine Anpassung des Registerharmonisierungsgesetzes vom 23. Juni 2006 (RHG; SR 431.02) möglich.

\section{Rechtspolitische Herausforderungen}

\section{1. Überforderung des politischen Systems?}

[43] E-Collecting soll das Unterschriftensammeln erleichtern und den gesamten Prozess vereinfachen (so etwa Motion Grüter). Aufgrund dieser Erleichterungen besteht die Befürchtung, E-Collecting könnte zu einer allzu grossen Anzahl von Volksabstimmungen führen und so das politische System überlasten (vgl. BisAz/SerdüLt 2017, 533 und 539; z. B. wird auch ein «NetzwerkEffekt» erwartet, insb. aufgrund der neuen sozialen Medien, vgl. Bisaz/SerdüLt 2017, 538). Es wird vor allem ein flächendeckendes Ergreifen des fakultativen Referendums gegen sämtliche Vorlagen und damit eine starke Verlangsamung des Gesetzgebungsprozesses befürchtet. So bestünde bei digitalen Plattformen die Möglichkeit von Mitnahmeeffekten, indem durch einen Klick in sehr kurzer Zeit mehrere Volksbegehren gleichzeitig unterstützt werden könnten (BIsAz/ SERDÜlt 2017, 539). Auch eine Lähmung des Parlaments durch eine Vielzahl parallel lancierter Volksinitiativen wird prognostiziert (vgl. BRAUn BINDER 2014, 556). Weiter wird davor gewarnt, dass die Meinungsbildung vor der Unterstützung von Volksbegehren qualitativ abnehmen könnte, wenn diese auf elektronischem Weg statt «im Dialog auf der Strasse» vor sich gehe (vgl. Bericht Civic Tech, 8). Daher wird flankierend zur Einführung des E-Collecting eine Anpassung der in der Verfassung festgelegten Quoren und Fristen für die Unterschriftensammlung diskutiert (BisAz/Serdült 2017, 540 f.). Dies liefe indes auf eine Erhöhung der Unterschriftenzahlen bei gleichzeitiger Verkürzung der Sammelfrist hinaus. 
[44] Gegenwärtig steht angesichts der fortdauernden Pandemie nicht die Überlastung des Gesetzgebungsprozesses, sondern im Gegenteil eine faktische Suspendierung der Volksrechte im Raum. Für die Bundesebene lassen sich wohl keine gesicherten Prognosen anstellen. Für die kantonale Ebene sind die Befürchtungen bezüglich einer Überlastung jedenfalls etwa am Beispiel des Kantons St. Gallen zu relativieren. Von 1953 bis 2020 sind im Kanton St. Gallen 59 kantonale Initiativen zustande gekommen, wovon 29 zur Abstimmung gelangt sind (vgl. Staatskanzlei St. Gallen 2020a). Von 1952 bis 2020 sind 39 Referendumsbegehren gültig zustande gekommen. Davon scheiterten 15 Vorlagen in der Referendumsabstimmung (vgl. Staatskanzlei St. Gallen 2020b). In den vergangenen knapp 70 Jahren wurde folglich circa jedes zweite Jahr über je eine Volksinitiative und ein Referendum abgestimmt. Damit ist das politische System des Kantons St. Gallen offensichtlich weit von einer Überlastungssituation entfernt. Ausserdem ist zu bedenken, dass in vielen Kantonen mittlerweile das von einer parlamentarischen Minderheit ergriffene Behördenreferendum die praktische Bedeutung des Volksreferendums stark mindert (GLASER/DöRIG 2020, 139 und 145). Im Kanton St. Gallen standen von 2010 bis 2020 vier Volksreferenden vier Behördenreferenden gegenüber.

\subsection{Deckelung des Anteils elektronisch gesammelter Unterschriften?}

[45] Um den Vorbehalten bezüglich der Überforderung des politischen Systems zu begegnen, wird eine Deckelung des Anteils elektronisch gesammelter Unterschriften vorgeschlagen (bspw. BisAz/Serdült 2017, 540 f.). Bei einer solchen Fixanteilslösung wird gesetzlich ein maximaler Anteil an Unterschriften festgelegt, der durch elektronische Unterstützungsbekundungen erbracht werden darf. Elektronische und herkömmliche Unterschriftensammlung kommen also komplementär zum Einsatz.

[46] Dabei ist offen, wie hoch der maximale Anteil an elektronischen Unterstützungsbekundungen sein soll. BisAz/Serdült sprechen von einem «vorerst geringen» Anteil (Bisaz/Serdült 2017, 543). Die St. Galler Regierung erwähnte einen Anteil von 30 Prozent (Antwort der Kantonsregierung auf Interpellation Louis/Schmid). Das E-Collecting würde somit lediglich das Erreichen des Unterschriftenquorums erleichtern. Daneben wäre weiterhin die Sammlung handschriftlicher Unterschriften erforderlich.

[47] Mag ein solcher Mechanismus für eine Pilot- oder Einführungsphase noch praktikabel sein, würde er doch nach einer Etablierung des E-Collecting zu überspitztem Formalismus führen. Den Stimmberechtigten ist zuzutrauen, Referenden und Volksinitiativen auf analogem wie auf digitalem Weg gleichermassen ernsthaft zu beurteilen und derartige Begehren nicht mutwillig zu unterzeichnen. Ähnlich wie heute beim Entscheid zwischen Stimmabgabe per Brief oder an der Urne sollte es den Stimmberechtigten zukünftig überlassen werden, ob sie ein Volksbegehren auf handschriftlichem oder elektronischem Weg unterstützen wollen. Daher sind auch Vorschläge abzulehnen, wonach die Initiativkomitees bei der Lancierung die abschliessende Entscheidung für entweder handschriftliche oder elektronische Unterstützungsbekundungen treffen müssten (Ammann/Schnell 2019, 33 f.). Analog zum Modus der Stimmabgabe vollzieht die Anpassung des Modus der Unterstützungsbekundung veränderte gesellschaftliche Rahmenbedingungen und Gewohnheiten nach. 
Dr. iur. des. Katja Gfeller, Juristische Mitarbeiterin am Bezirksgericht Frauenfeld, E-Mail: gfeller.k@gmail.com.

Prof. Dr. iur. Andreas Glaser, Inhaber des Lehrstuhls für Staats-, Verwaltungs- und Europarecht an der Universität Zürich unter besonderer Berücksichtigung von Demokratiefragen; Mitglied der Direktion des Zentrums für Demokratie Aarau (ZDA), E-Mail: andreas.glaser@zda.uzh.ch.

MLaw, LL.M. Irina Lehner, Doktorandin am Zentrum für Demokratie Aarau, E-Mail: irinaesther.lehner@zda.uzh.ch.

Interessenbindung:

Der Beitrag beruht auf einem Rechtsgutachten des Zentrums für Demokratie Aarau (ZDA) im Auftrag der Staatskanzlei des Kantons St. Gallen aus dem Jahr 2018.

\section{Literatur- und Materialienverzeichnis}

Ammann, Matthias/Schnell, Fabian (2019): Digitale Demokratie - Schweizer Volksrechte stärken, Zürich.

Biaggini, Giovanni (2020): Der coronavirusbedingte Fristenstillstand bei eidgenössischen Volksbegehren: eine Fallstudie zur Tragfähigkeit von Art. 185 Abs. 3 BV, in: ZBl 121/2020, S. 277 ff.

Bisaz, Corsin/Serdült, Uwe (2017): E-Collecting als Herausforderung für die direkte Demokratie der Schweiz, in: LeGes 28 (2017) 3, S. $532 \mathrm{ff}$.

BRAUn Binder, NADJA (2014): Quoren und Fristen bei der elektronischen Unterschriftensammlung (E-Collecting), in: ZSR 2014, S. $539 \mathrm{ff}$.

Braun, NADJA (2006): Stimmgeheimnis. Eine rechtsvergleichende und rechtshistorische Untersuchung unter Einbezug des geltenden Rechts, Diss. Bern.

Bundeskanzlei (2020): Initiativen und Referenden unterschreiben - trotz Corona https://www.admin.ch/gov/de/start/ dokumentation/abstimmungen/unterschriftensammlungen.html [18.12.2020].

Bundesrat (2002): Bericht über den Vote électronique vom 9. Januar 2002, Chancen, Risiken und Machbarkeit elektronischer Ausübung politischer Rechte, BB1 2002645 ff. (zit. Bericht Vote électronique 2002).

Bundesrat (2009): Stellungnahme vom 18. Februar 2009 zur Motion Fehr (08.3908), Stärkung der Demokratie durch E-Collecting vom 18. Dezember 2008 https://www.parlament.ch/de/ratsbetrieb/suche-curia-vista/geschaeft?AffairId= 20083908 [18.12.2020] (zit. Stellungnahme des Bundesrats zur Motion Fehr).

Bundesrat (2012): Stellungnahme vom 16. Mai 2012 zur Interpellation Wermuth (12.3082), Zentrale Beglaubigung für eidgenössische Volksinitiativen und Referenden vom 7. März 2012, https://www.parlament.ch/de/ratsbetrieb/suchecuria-vista/geschaeft?AffairId=20123082 [18.12.2020] (zit. Stellungnahme des Bundesrats zur Interpellation Wermuth).

Bundesrat (2013): Bericht über den Vote électronique vom 14. Juni 2013, Auswertung der Einführung von Vote électronique (2006-2012) und Grundlagen zur Weiterentwicklung, BB1 20135069 ff. (zit. Bericht Vote électronique 2013).

Bundesrat (2017): Medienmitteilung vom 5. April 2017, Bundesrat beschliesst nächste Schritte zur Ausbreitung der elektronischen Stimmabgabe https://www.admin.ch/gov/de/start/dokumentation/medienmitteilungen.msg-id-66273.html [18.12.2020] (zit. Medienmitteilung 2017).

Bundesrat (2018): Botschaft zum Bundesgesetz über elektronische Identifizierungsdienste vom 1. Juni 2018, BB1 20183915 (zit. Botschaft BGEID 2018).

Bundesrat (2018): Stellungnahme vom 16. Mai 2018 zur Motion Grüter (18.3062), Stärkung der Volksrechte. Unterschriftensammlung für Initiativen und Referenden im Internet vom 5. März 2018 https://www.parlament.ch/de/ratsbetrieb/ suche-curia-vista/geschaeft?AffairId=20183062 [18.12.2020] (zit. Stellungnahme des Bundesrats zur Motion Grüter). 
Bundesrat (2019): Botschaft zur Änderung des Bundesgesetzes über die Alters- und Hinterlassenenversicherung (Systematische Verwendung der AHV-Nummer durch Behörden) vom 30. Oktober 2019, BBl 20197359 (zit. Botschaft Änderung AHVG 2019).

Bundesrat (2020): Bericht in Erfüllung der Postulate 17.3149 Hausammann Markus und 17.4017 Müller Damian vom 8. Mai 2020, Civic Tech und Vereinfachung des Vernehmlassungsverfahrens: Entwicklungen und Massnahmen https://www.bk.admin.ch/bk/de/home/dokumentation/e-government/civictech.html [18.12.2020] (zit. Bericht Civic Tech).

Bundesrat (2020): Medienmitteilung vom 20. März 2020, Coronavirus: Sammel- und Behandlungsfristen von eidgenössischen Volksbegehren stehen still https://www.admin.ch/gov/de/start/dokumentation/medienmitteilungen.msg-id78503.html [18.12.2020] (zit. Medienmitteilung 2020).

E-Government Schweiz (2020): Jahresbericht 2019 https://www.egovernment.ch/de/dokumentation/jahresberichte/ [18.12.2020].

Eidgenössischer Datenschutz- und Öffentlichkeitsbeauftragter (2019): Leitfaden Datenschutz bei Wahlen und Abstimmungen https://www.edoeb.admin.ch/edoeb/de/home/datenschutz/dokumentation/leitfaeden/Wahlen.html [18.12.2020] (zit. EDÖB 2019).

Gerny, Daniel (2020): Keine Volksinitiative seit März: Das Coronavirus schnürt den Schweizer Volksrechten die Luft ab, in: Neue Zürcher Zeitung vom 29. August 2020 https://www.nzz.ch/schweiz/wie-das-coronavirus-den-schweizervolksrechten-die-luft-nimmt-ld.1573728 [18.12.2020].

Gfeller, Katja/Braun Binder, Nadja/Serdült, Uwe (2019): Demokratie im digitalen Zeitalter. Das Beispiel von Initiative und Referendum in der Schweiz, in: Kübler, Daniel/Glaser, Andreas/Waldis, Monika (Hrsg.), Brennpunkt Demokratie: 10 Jahre Zentrum für Demokratie Aarau, Baden, S. 48 ff.

Glaser, Andreas/Dörig, Heidi (2020): Wahlrecht und direkte Demokratie unter der Kantonsverfassung von 1980 - Entwicklungen und Reformbedarf, in: Ziegler, Béatrice/Glaser, Andreas (Hrsg.), Direkte Demokratie im Kanton Aargau in Geschichte und Gegenwart, Zürich, S. $139 \mathrm{ff}$.

Informatik Kanton und Stadt Schaffhausen (2019): Schaffhauser eID+ https://sh.ch/CMS/Webseite/Kanton-Schaffhausen/Beh-rde/Services/Schaffhauser-eID-2077281-DE.html [18.12.2020].

Interpellation Louis-Nesslau/Schmid-Grabs (51.18.22): Einführung von E-Collecting im Kanton St. Gallen, Interpellation Kantonsräte Ivan Louis/Sascha Schmid vom 23. April 2018 https://www.ratsinfo.sg.ch/geschaefte/3588\#documents [18.12.2020] (zit. Interpellation Louis/Schmid).

Kantonsregierung St. Gallen (2018): Antwort vom 29. Mai 2018 auf Interpellation Louis/Schmid (51.18.22), Kanton St. Gallen als E-Collecting-Pionier vom 23. April 2018 https://www.ratsinfo.sg.ch/geschaefte/3588\#documents [18.12.2020] (zit. Antwort der Kantonsregierung auf Interpellation Louis/Schmid).

Motion Fehr (08.3908): Stärkung der Demokratie durch E-Collecting, Motion Nationalrätin Jacqueline Fehr vom 18. Dezember 2008 (zit. Motion Fehr).

Motion Grüter (18.3062): Stärkung der Volksrechte. Unterschriftensammlung für Initiativen und Referenden im Internet, Motion Nationalrat Franz Grüter vom 5. März 2018 (zit. Motion Grüter).

Staatskanzlei St. Gallen (2020a): Kantonale Initiativen seit 1953 https://www.sg.ch/politik-verwaltung/abstimmungenwahlen/initiativen—referenden.html [18.12.2020].

Staatskanzlei St. Gallen (2020b): Kantonale Referenden seit 1952 https://www.sg.ch/politik-verwaltung/abstimmungenwahlen/initiativen—referenden.html [18.12.2020].

Stiftung für direkte Demokratie (2020): Offener Brief an Bundespräsidentin Simonetta Sommaruga vom 28. August 2020, COVID-19 bedroht unsere direkte Demokratie https://www.demokratie.ch/blog/offener-brief-an-bundesprasidentinsimonetta-sommaruga-covid-19-bedroht 18.12.2020 [18.12.2020].

Volksmotion Schaffhausen (2020/1): Mehr Demokratie in Schaffhausen - einfach und sicher: Volksbegehren auch elektronisch unterschreiben (E-Collecting) https://sh.ch/CMS/Webseite/Kanton-Schaffhausen/Beh-rde/Parlament/DerKantonsrat/Portal-Kantonsrat—Ratsbetrieb-2307955-DE.html [18.12.2020] (zit. Motion Schaffhausen).

von Matt, Ottmar (2020): «Coronakrise deckte zwei grosse Versäumnisse der Schweiz auf»: Aktivisten kämpfen für modernere Demokratie, in: Aargauer Zeitung vom 4. Mai 2020 https://www.aargauerzeitung.ch/schweiz/corona krise-deckte-zwei-grosse-versaeumnisse-der-schweiz-auf-aktivisten-kaempfen-fuer-modernere-demokratie-137778329 $[18.12 .2020]$. 